\title{
Arrays of pentacene single crystals by stencil
}

\section{evaporation}

Pavlo Fesenko ${ }^{1,2^{*}}$, Valentin Flauraud ${ }^{3}$, Shenqi Xie ${ }^{3}$, Jürgen Brugger ${ }^{3}$, Jan Genoe $^{1,2}$, Paul

Heremans $^{1,2}$, Cédric Rolin ${ }^{1}$

${ }^{1}$ imec, Large Area Electronics, Kapeldreef 75, 3001 Leuven, Belgium

${ }^{2}$ KU Leuven, Department of Electrical Engineering, Kasteelpark Arenberg 10, 3001, Leuven, Belgium

${ }^{3}$ EPFL, Microsystems Laboratory 1, Station 17, CH-1015 Lausanne, Switzerland

*Corresponding author. Tel.: +32 162833 53. E-mail address: pavlo.fesenko@imec.be

Keywords: organic semiconductors, pentacene, single crystals, stencil evaporation, diffusion. 


\section{Supporting Information}
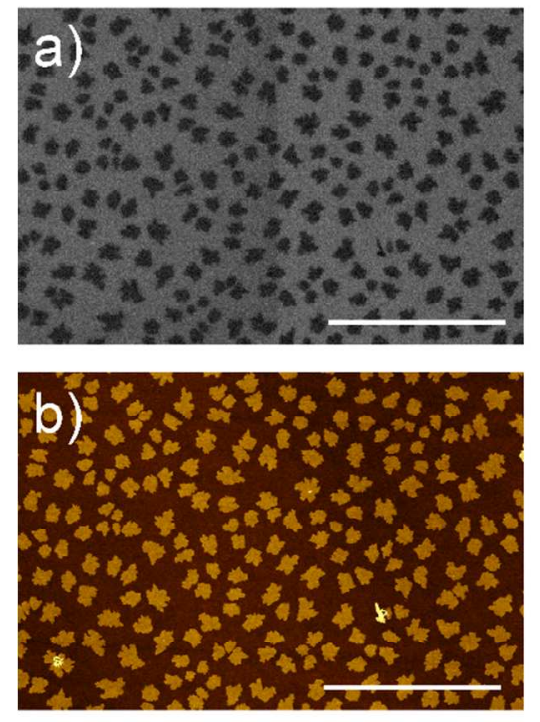

c)

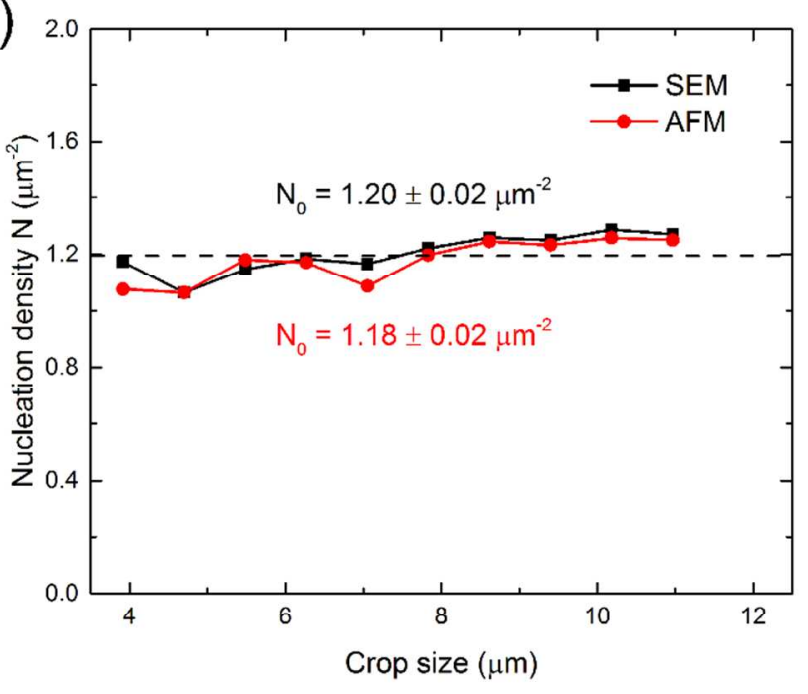

Figure S1. Nucleation densities $N$ of the pentacene submonolayer film for different crop sizes calculated from the a) SEM and b) AFM images of the same area. The scale bars on all images are $5 \mu \mathrm{m}$. 


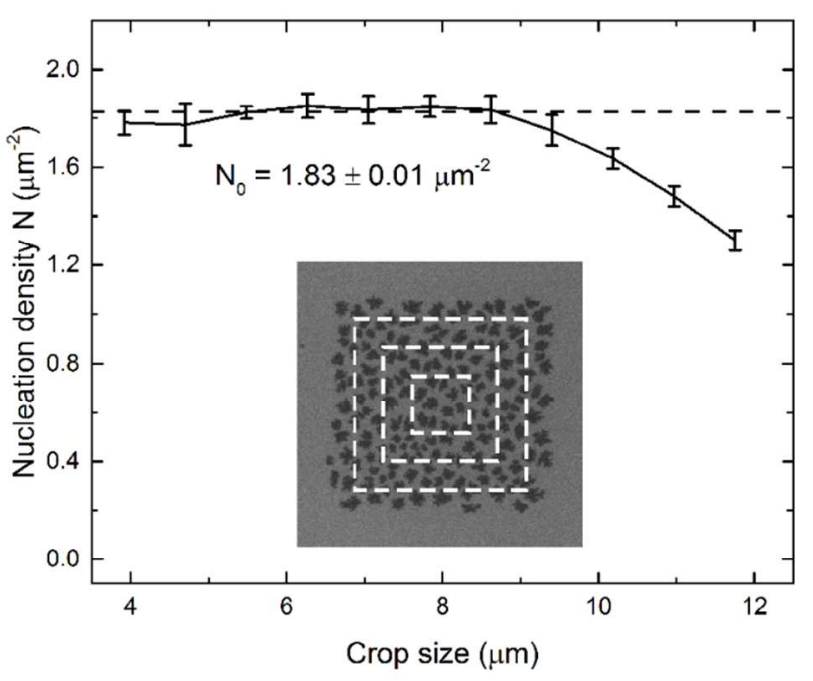

Figure S2. Nucleation density $N$ of pentacene submonolayer film for the aperture $a=10 \mu \mathrm{m}$ as a function of the SEM image crop size. The background nucleation density $N_{0}=1.83 \pm 0.01 \mu \mathrm{m}^{-2}$ is calculated from the nucleation density unaffected by diffusion losses along the perimeter of the aperture. The SEM image is shown in the inset.

a)

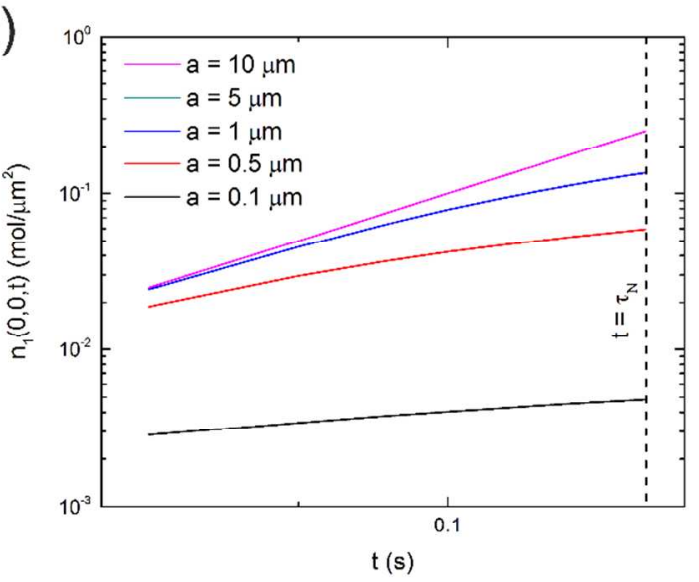

b)

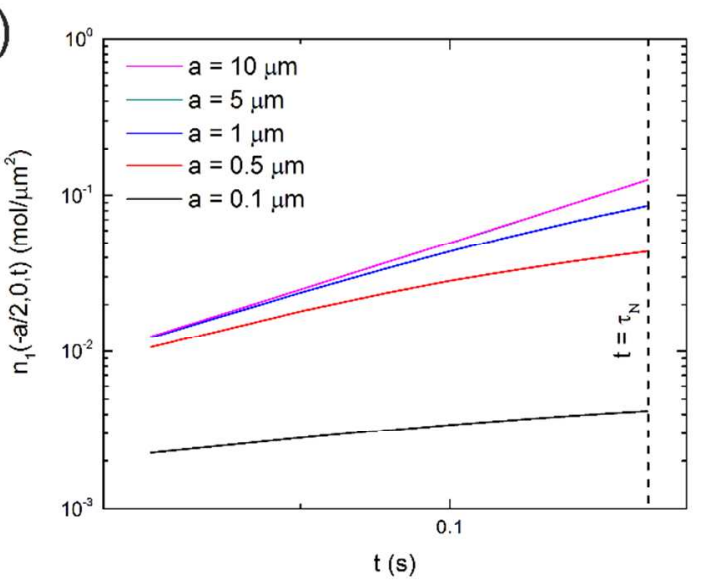

Figure S3. Molecular concentration in the middle of the aperture $n_{1}(0,0, t)$ and at the edge $n_{1}(-$ $\mathrm{a} / 2,0, t)$ as a function of $t$ for different aperture sizes $a$. The simulation parameters are $D=1$ 
$\mu \mathrm{m}^{2} / \mathrm{s}, \tau_{\mathrm{N}}=0.25 \mathrm{~s}$ that correspond to $\lambda_{\mathrm{N}}=0.5 \mu \mathrm{m}$. The time to nucleation $\tau_{\mathrm{N}}$ is marked by the dashed line. 